\title{
Nondestructive Determination of Fruit Based on Sensory and Near-Infrared Technology
}

\author{
Qionglei LIU ${ }^{1,2, a}$, Baohua TAN $N^{1,2, b^{\star}}$
}

\author{
${ }^{1}$ Hubei Collaborative Innovation Center for High-efficiency Utilization of Solar Energy, Huei \\ University of Technology, Wuhan, 430068, China \\ ${ }^{2}$ School of Science, Hubei University of Technology, Wuhan, 430068, China \\ aemail: 2269931044@qq.com, bemail: tan_bh@126.com, "corresponding author
}

Key words: subjective analysis, objective analysis, near-infrared technology

\begin{abstract}
It can be divided into subjective and objective analysis on the sensory analysis of fruits. Subjective analysis almost based on sight, smell, touch, hearing and sense of taste, and the information of fruits will be analyzed when it was transmitted to brain after sensory cell receiving external stimuli. Objective analysis refers to the intelligent detection combined with modern technology, and the paper is mainly about fruit quality analysis based on near-infrared analytical technique. Otherwise, the differences and combinations between two analysis were also introduced.
\end{abstract}

\section{Introduction}

Fruits like apple, kiwi and watermelon are one of the most popular and the most important cash fruits in the world. China is one of the world's fruit-producing countries, and fruit tree species in China are very rich in resources, the main fruit and dried fruit has more than 50 species. Although fruit output ranked top, fruit exports accounted for only about $2 \%$ of total output, the main reason of that is the lagging fruit post-harvest processing technology. Near-infrared technology has become a well-accepted method for the assessrnent of fruit with the development of optical technology and stoichiometry. And sensory analysis method and NIRS have been introduced below. The distinction of subjective analysis method (sensory analysis) and objective analysis (NIRS) were also listed.

\section{Subjective Analysis based on Senses}

Vision. Vision is a kind of subjective sensation, which is through the visual system of peripheral organs (eyes) to accept the external environment in a certain wavelength range of electromagnetic stimulation and then encoded and analyzed by the central departments. The maturity and damage of fruit can be identified when we look at the surface through the vision. We can visually identify that the apple in Fig. a is fresh and ripe, and the apple in Fig. b is obviously rotten (Fig. 1). So we can identify the quality of the fruit through the vision.

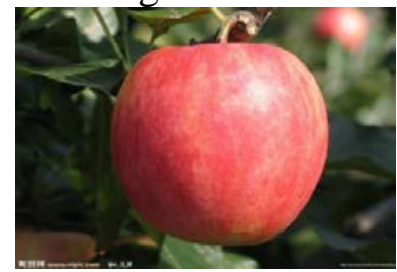

(a)

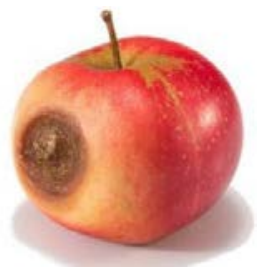

(b)

Fig. 1 Comparison of different quality of apples

(Picture Source: http://www.nipic.com/show/1/42/5750190k8561e040.html)

Smell. Smell is a sense of chemical stimulation through the long-distance which olfactory nervous system and the nasal trigeminal nervous system are participated. On the one hand, We can't judge just depend on smell because there are many things inaccurate. For example, some fruits don't have enough fruity to distinguish its quality. On the other hand, we can smell the pesticide when fruits were sprayed the pesticide, which we can't judge by other organs. So the smell is mainly to play an auxiliary role, but it is also essential. 
Touch. Touch refers to a feeling that nerve cells in the body's skin feel the temperature, humidity, pain, pressure, vibration and other aspects from the outside world. It is the sensation of biological contact, especially the feeling of contact surface stimulation. It is caused by the pressure and traction on the touch receptors. We can identify fruit's hardness, frangibility and other sensory indicators with touching fruit. We can touch kiwi to distinguish its hardness and identify its maturity (Fig. 2).

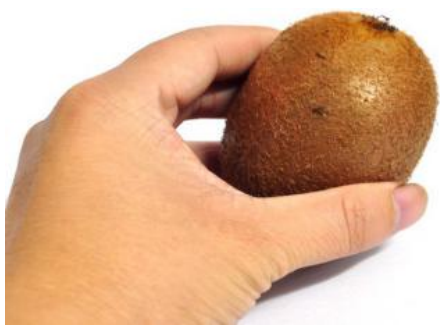

Fig. 2 Kiwi kneaded by hand

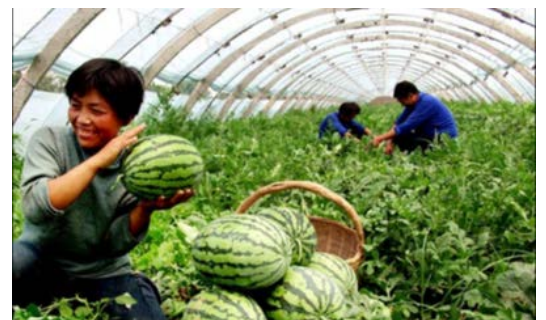

Fig. 3 Distinguish watermelon maturity

(Fig. 2 Picture Source: http://photo.redocn.com/diet/2013-11-14/2406008.html)

(Fig. 3 Picture Source: http://www.biyi.net/list/100/689898.html)

Auditory Sense. Sensory cells were excited when sound waves act on the auditory organs, and then the cells will deliver the impulse of the auditory nerve, and auditory sense comes when delivered information was analyzed by the hearing center. We can listen to the sound of watermelon by tapping it to judge its mature as shown in Fig. 3. In addition, we can also listen to the sound of masticating fruit to determine its quality, such as eating melon or apple. And the melon is still fresh if you hear the crunchy sound.

Taste. Taste refers to a feeling caused by food in the oral cavity, and it is also an ability to feel the taste of a material. Tongue in Fig. a is the taste buds (Fig. b), and there are many taste-sensing molecules on the surface of gustatory. Different substances can be combined with different taste receptors and show different taste, and sensitive map (Fig. c) of sour, sweet, bitter and salty are also in different position. Taste is one of the most direct way of feeling, and we can identify the fresh of the fruit by just eating. Some fruits do not change the surface after the deterioration, but the taste will always be different. So we can determine whether the fruit is spoiled through the taste.

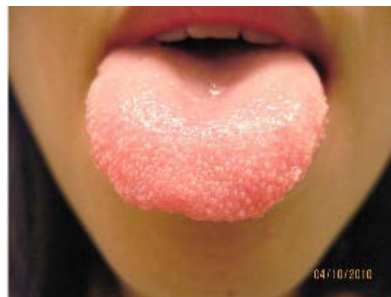

(a)

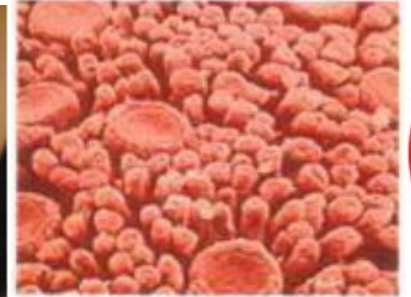

(b)

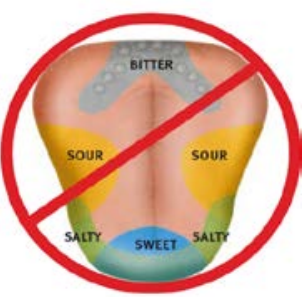

(c)

Fig. 4 Taste buds

(Picture Source: http://www.jscdc.org/Article3-2268-2-0.aspx)

\section{Objective Analysis based on Near Infrared Technology}

Near Infrared Spectrometer. Near infrared spectroscopy is of overtone vibrational spectroscopy behaving in the near infrared region of $780-2500 \mathrm{~nm}$ where overtones or combinations of fundamental stretching bands response [1]. Spectra in this region contain abundant information reflecting the structure of molecules as well as attributes of objects such as firmness, granularity, etc. For these bands are reflected bonds of $\mathrm{C}-\mathrm{H}, \mathrm{N}-\mathrm{H}$, and $\mathrm{O}-\mathrm{H}$ (bonds with hydrogen atoms tend to vibrate independently from the rest part [2]), analysis of organic molecules under the use of near infrared absorption is feasible [3]. The energy absorption of organic molecules in NIR region occurs when molecules vibrate is translated into an absorption spectrum within the NIR spectrometer [4]. The near-infrared absorption spectra can be correlated with the component or property data of the measured substance by means of appropriate stoichiometry, and then a corresponding model can be established. 


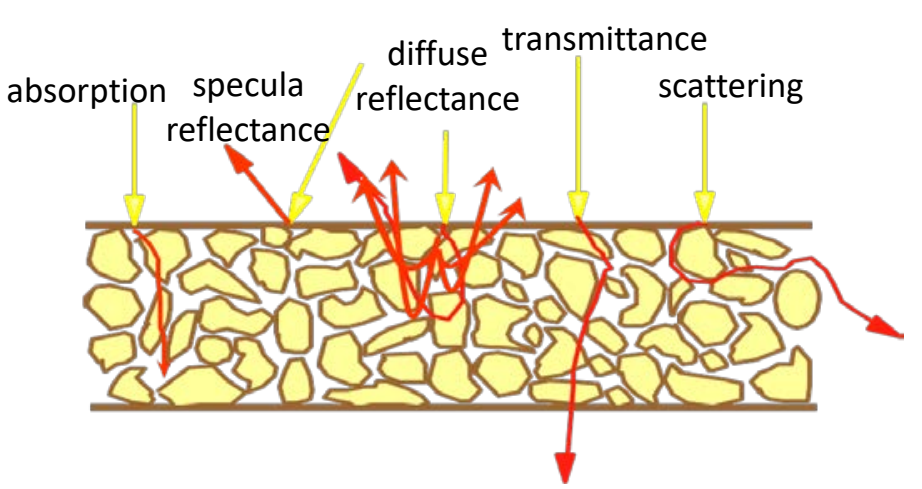

Fig. 5 The interaction of Near-infrared light and substance

There are five situations when the near-infrared light is irradiated on the object(Fig. 5): (1) completely absorbed; (2) total reflection; (3) diffuse reflection; (4) transmission; (5)scattering. In the interaction of near-infrared light and substance, the structural information of substance can be obtained by transmission and diffuse reflection, and the density of substance can be determined by total reflection.

Near-Infrared Spectroscopy Analysis of Technical Characteristics and Limitations. Near-infrared spectroscopy (NIRS) is a modern and emerging indirect analytical technique combining spectroscopy and chemometrics. It is a qualitative or quantitative analysis of unknown samples through the establishment of calibration models. It is better than other detection methods, and its advantages can be summarized as following: (1) rich information: to be able to get a large number of groups of structural information, fully reflect the characteristics of the measured material; (2) simple and fast analysis process: the near-infrared spectroscopy technology is analyzed by computer processing, so its speed is very fast, and the whole process will not exceed 2 minutes [5]; (3) non-destructive testing and green security; (4) a variety of measuring ways: transmission, reflection and diffuse reflection; (5) more accurate results.

NIR spectroscopy analysis is widely utilized in fruit quality assessment these years. However, some problems should be in-depth solved for ongoing/future research. First, the poor characteristic of the near-infrared spectrum of the material results in the dependence of the analytical technique on chemometrics. Second, the similarity of the substance's near-infrared spectroscopy results in the need of highly stable near-infrared spectrometer, which is difficult for the requirement of domestic production technology. Third, near-infrared spectroscopy depends too much on the mathematical model established by stoichiometric method. But the adaptability of the original mathematical model is deteriorated, and there is not a good mathematical model transfer method for now.

Nondestructive Determination of Fruit Through Near-Infrared Technology. Near Infrared (NIR) analysis mainly consists of two processes: the calibration process and the forecasting process. The left side is the process for the establishment of the model, the right is the process of forecasting model (Fig. 6). Thus, the near-infrared spectroscopy mainly includes three parts: spectrometer, stoichiometry and model. The combination of these three indispensable parts make the analysis results tested fast and accurately.

Dong detect the quality of apples using NIRS, and hardness, SSC, MC, and $\mathrm{pH}$ of apples were analyzed by different models in the paper. The SPA-LSSVM model had better comprehensive ability in determining SSC, MC, and pH than others, with the correlation coefficient of prediction of $0.961,0.984$, and 0.882 and residual predictive deviation of 3.49, 5.51, and 2.06, respectively [6]. The results demonstrated the feasibility of using near-infrared hyperspectral reflectance imaging technology as a non-invasive method for predicting SSC, MC, and pH of apples simultaneously. 


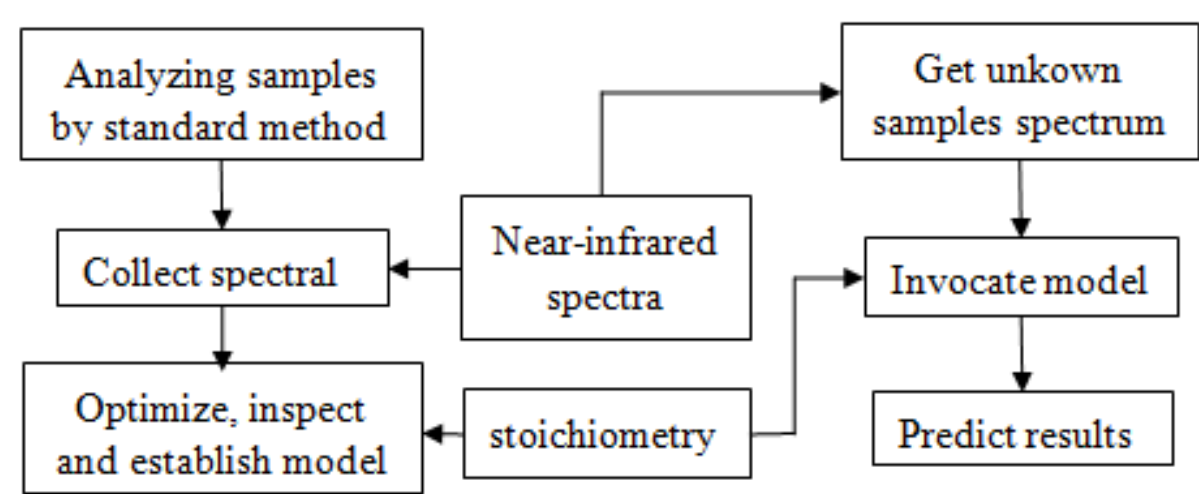

Fig. 6 Near-infrared analysis process

There is also research about detecting grapes quality using NIRS. Least-Squared Support Vector Machine (LS-SVM) regression, a semi-parametric modeling technique, is used to predict the acidity of three different grape varieties using NIR spectra. The performance and robustness of LS-SVM regression are compared to Partial Least Square Regression (PLSR) and Multivariate Linear Regression (MLR). LS-SVM regression produces more accurate prediction [7].

Calibration models were built to test SSC, DM, TA, PF of mango using PLS method. Coefficient of determination and root mean square errors of prediction (RMSEP) values were, respectively: 0.92 and 0.55 Brix for SS, 0.67 and $0.51 \%$ for DM, 0.50 and $0.17 \%$ citric acid for TA, 0.72 and 12.2 $\mathrm{N}$ for $\mathrm{PF}$ [8]. And the results show that it is feasible to determine quality parameters in the 'Tommy Atkins'mango using the new NIR spectrometer.

\section{The Difference and Combination between NIR and Senses}

The Difference in Life. The maturity, damage and freshness of fruit can be identified through the five sense organs in daily life. As long as we have enough life experience, we will generally find the fruit in good quality. However, there are also some fruits which is not easy to identify its maturity such as cantaloupe, pineapple, durian, watermelon, etc.

The maturity of fruit can be identified by testing soluble solids content, the $\mathrm{PH}$ value and hardness using NIRS. It is a nondestructive determination for fruit quality in Fig. 7, which only needs to detect the surface of the fruit with the head contact, and it can simultaneously test the SSC, total acidity, maturity, hardness, color, water content index and so on. And the tested value are displayed on the LCD screen after only 2 seconds. But these products are mostly from abroad, and the price is very high, most of these are used in some agricultural research at present.

we are still more accustomed to judging the maturity of fruit by the sensory now. But with the continuous development of NIR, the cost of nondestructive determination for fruit quality will be lower, and its application will become more and more widely in daily life.

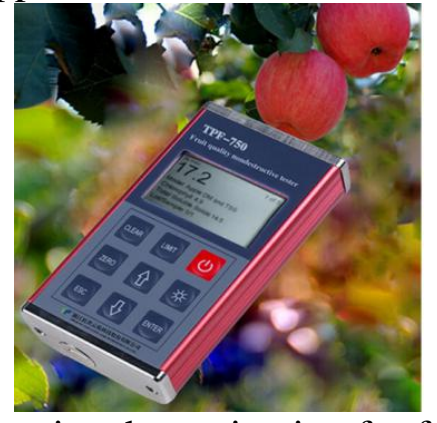

Fig. 7 Nondestructive determination for fruit quality

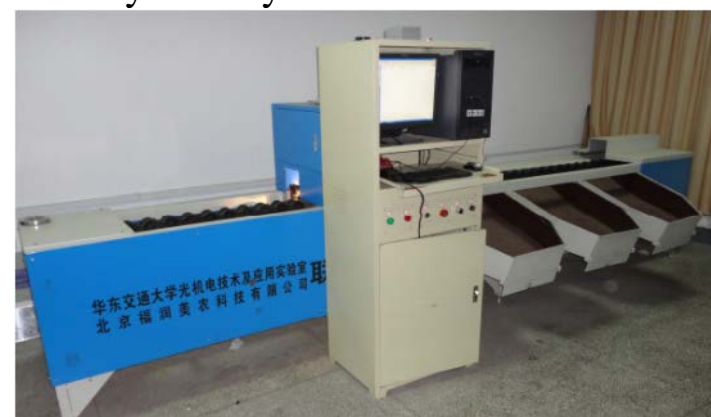

Fig. 8 On - line detection system of fruit

(Picture Source: http://www.top17.net/product/2873.html)

The Difference of Industry. Traditional way of classification like artificial classification is to identify the hardness, the damaged condition, the maturity and the fresh of fruit by people. When this kind of classification was used on the identification of a large number of fruit, it will be time-consuming and the results will also not be very accurate.

New near-infrared spectroscopy is a non-destructive, environmental friendly, rapid technique 
capable for on-line application. NIR spectroscopy's on-line application in fruit quality assessment of industrial use have been invented one after another. And the near-infrared on-line detection device will automatically help to separate the fruit of different quality and screen out the bad one to avoid deterioration of other fruits, which is more efficient and faster than the traditional way. The near-infrared spectroscopy on-line nondestructive testing equipment (Fig. 8 [9]) is a highly efficient, accurate and quick equipment, it can detect precisely three per second. Foreign technology in this field is more mature now, and the device are relatively more precise and stable too.

Traditional way of classification like artificial classification is, however, labour-intensive, time-consuming, and expensive. New near-infrared on-line detection offer a straight-forward, rapid, and cost effective alternative. Because of immature technology and high cost, even if it has many benifits, but also not universal.

Near Infrared Technology Combined with Sensory Analysis. Sensory analysis is a scientific and objective method combined with psychological, physical, statistics and other disciplines, with the help of the human sensory organs (auditory, olfactory, taste, visual, touch) to evaluate, measurement, analysis and interpret the sensory properties of food, to proceed qualitative and quantitative analysis of the food, to understand the feelings of people for these products and detect the characteristics of quality of the product itself [10].

Sensory analysis combined with near-infrared technology refers to detect and analyze the food with the help of near-infrared technology instead of the human sensory. The changes in organoleptic properties of most food products arise from changes in their chemical composition [11]. For example, the hardness and the size of fruit is closely related to the content of pectin, and we can get the information of hardness by just detecting the content of fruit [12]. Therefore, we can analyze fruit acidity, sweetness, hardness, internal browning, surface damage and other indicators using the near-infrared technology.

With the development of electronic technology, optics, chemometrics and computer technology, near infrared analysis technology has been paid more and more attention in the field of fruit quality detection. Fruit detection on NIRS has already made some great achievement abroad, and those technology has also been put into production, but there still are many deficiencies. Many domestic scholars are also continuing to study, and strive to find a more stable model.

\section{Summary}

Near infrared spectroscopy technology has been extensively and effectively employed in a variety of fields both for research and application utilities, including areas of food, agricultural, chemical, polymers, cosmetics and medical etc., though inherent limiting factors it might have. For on-line application, the potentials are limited by the high price of NIR spectroscopy. In addition, the stability and accuracy of the model need to be improved. And the correlation between near-infrared spectroscopy and food sensory index also needs further study.

\section{Acknowledgment}

This work is supported by the College student's research projects of science and technology innovation fund of the Hubei Collaborative Innovation Center for High-efficiency Utilization of Solar Energy (NO.HBSDY201503), and the College students' innovation and Entrepreneurship training plan of the national education department (NO. 201510500003).

\section{References}

[1] Lin H, Ying Y. Theory and application of near infrared spectroscopy in assessment of fruit quality: a review[J]. Sensing and instrumentation for food quality and safety, 2009, 3(2): 130-141.

[2] Hruschka W R, Williams P, Norris K. Near infrared technology in the agricultural and food industries[J]. Data analysis: Wavelength selection methods. American Association of Cereal 
Chemists, 2001.

[3] Stuart B. Infrared Spectroscopy: Fundamentals and applications, 2004 John Wiley \& Sons[J]. Ldt, 33(35): 59-62.

[4] Cen H, He Y. Theory and application of near infrared reflectance spectroscopy in determination of food quality[J]. Trends in Food Science \& Technology, 2007, 18(2): 72-83.

[5] Dalmay T, et al. Replication of Cymbidium ringspot virus satellite RNA mutants[J]. Virology, 1995,206(2): 1 092-1 098.

[6] Dong J, Guo W. Nondestructive determination of apple internal qualities using near-infrared hyperspectral reflectance imaging[J]. Food Analytical Methods, 2015, 8(10): 2635-2646.

[7] Chauchard F, Cogdill R, Roussel S, et al. Application of LS-SVM to non-linear phenomena in NIR spectroscopy: development of a robust and portable sensor for acidity prediction in grapes[J]. Chemometrics and Intelligent Laboratory Systems, 2004, 71(2): 141-150.

[8] Marques E J N, de Freitas S T, Pimentel M F, et al. Rapid and non-destructive determination of quality parameters in the 'Tommy Atkins' mango using a novel handheld near infrared spectrometer[J]. Food chemistry, 2016, 197: 1207-1214.

[9] GAO Ronjie, Study on vis/near infrared spectroscopy for dynamic online nondes tructive detecting sugar content in fruits[D]. East China Jiaotong University, 2012.

[10]ZHU J, LV F. Development of study on sensory evaluation in food [J][J]. China Condiment, 2009, 5: 003.

[11]LUO Y, WANG X, DENG D. Near Infrared Spectroscopy Technology and Its Application in Food Sensory Analysis [J][J]. Food Science, 2009, 7: 064.

[12] McGlone V A, Jordan R B, Martinsen P J. Vis/NIR estimation at harvest of pre-and post-storage quality indices for 'Royal Gala'apple[J]. Postharvest Biology and Technology, 2002, 25(2): 135-144. 